\title{
OFFENE STANDARDS UND VERTEILTE ANWENDUNGEN ALS GRUNDLAGE „VERTEILTER WISSENSARBEIT“ (AUCH) IM OPEN GOVERNMENT
}

\author{
Maika Büschenfeldt, Margit Scholl
}

\section{Zusammenfassung}

Im Beitrag wird die Frage behandelt, inwieweit und in welcher Form offene Standards und verteilte Anwendungen als Grundlage einer „verteilten Wissensarbeit“ wirksam werden. Verteilte Wissensarbeit wiederum wird als substantielle Grundlage zur Beherrschung komplexer Herausforderungen und Problemlagen sowie als Voraussetzung für Innovationen und zur Schaffung von Mehrwert gefasst. Wir entwickeln unsere grundsätzliche Position wissenschaftstheoretisch und reflektieren sie an den aktuellen Zielen, Prozessen und Beispielen von Open Government, ohne sie darauf zu begrenzen.

\section{Abstract}

The article addresses the question of how far and how 'open standards' and distributed applications take effect as the basis of a "distributed knowledge work". Distributed knowledge work itself is taken as a substantial basis for managing the complexity of current challenges and to create value. We develop our fundamental position epistemologically and reflect the current goals, processes and examples of open government, but without limiting it.

\section{»I. EINLEITUNG: OFFENE/VERTEILTE WISSENSARBEIT}

Der soziologische Begriff der ,Wissensgesellschaft ${ }^{11}$ beschreibt die ökonomische Dimension des gesellschaftlichen Wandels von der industriellen Gesellschaft zur postindustriellen Gesellschaft. Als Indiz dieses Wandels wird die erhebliche Bedeutungszunahme des Wissens als wichtigste Ressource und als „Produktionsfaktor“ gedeutet. Wissen steht in Begriff, die klassischen Produktivkräfte - Land, Boden und Kapital in ihrer ökonomischen Bedeutung zu überflügeln (Willke 2001). Die Wissensgesellschaft ist zwar noch nicht erreicht, deutet sich aber bereits an: So zeichnet sich der Trend ab, dass der Markt zusehends von Produkten beherrscht wird, deren Wert auf der eingebauten Expertise beruht. Ein inzwischen historisches Beispiel ist der Intel 486 Mikroprozessor dessen Produktionskosten 1990 rund 100 Dollar betrugen, der aber für 500 Dollar verkauft wurde. Diese große Differenz zwischen Produktionskosten und Verkaufspreis lassen sich als Wissensabgabe deuten. $^{2}$

Die wachsende Bedeutung der Ressource Wissen wirft die Frage auf, auf welche Art und Weise mit Wissen künftig umzugehen ist und wie die Verbreitung, Nutzung und Generierung von Wissen gesteuert werden kann. Es geht insbesondere um die Kontrolle des Wissens, d. h. um die Regelung des Zugriffs auf Wissen und der Entscheidung, ob Wissen als eine Ware und damit als Grundlage des geistigen Eigentums zu betrachten sei oder, ob Wissen notwendig als ein öffentliches Gut behandelt werden muss (Grassmuck 2004). In dieser Frage stehen sich zwei Interessen und Positionen gegenüber:
Die erste Position rückt den Eigentumsschutz des Wissens als Urheber- und Investitionsschutz in den Mittelpunkt: Wissensschutz dient dazu, Wettbewerbsvorteile und Innovation zu sichern. Die nötigen Investitionen für Innovationen können in dieser Argumentation nur getätigt werden, wenn das gewonnene Wissen wirksam geschützt werden kann. Wissen kann durch die „falsche Verbreitung deutlich an Wert verlieren" (Clases und Wehner 2002: 51).

Die zweite Position betont das gesellschaftliche Interesse an der freien Verfügbarkeit des Wissens. Wissensfreiheit wird in dieser Argumentation als Garant für Innovation und Forschung gesehen. Innovation braucht immer auch ein gewisses Maß an Offenheit (z. B. bei Grassmuck 2004). 


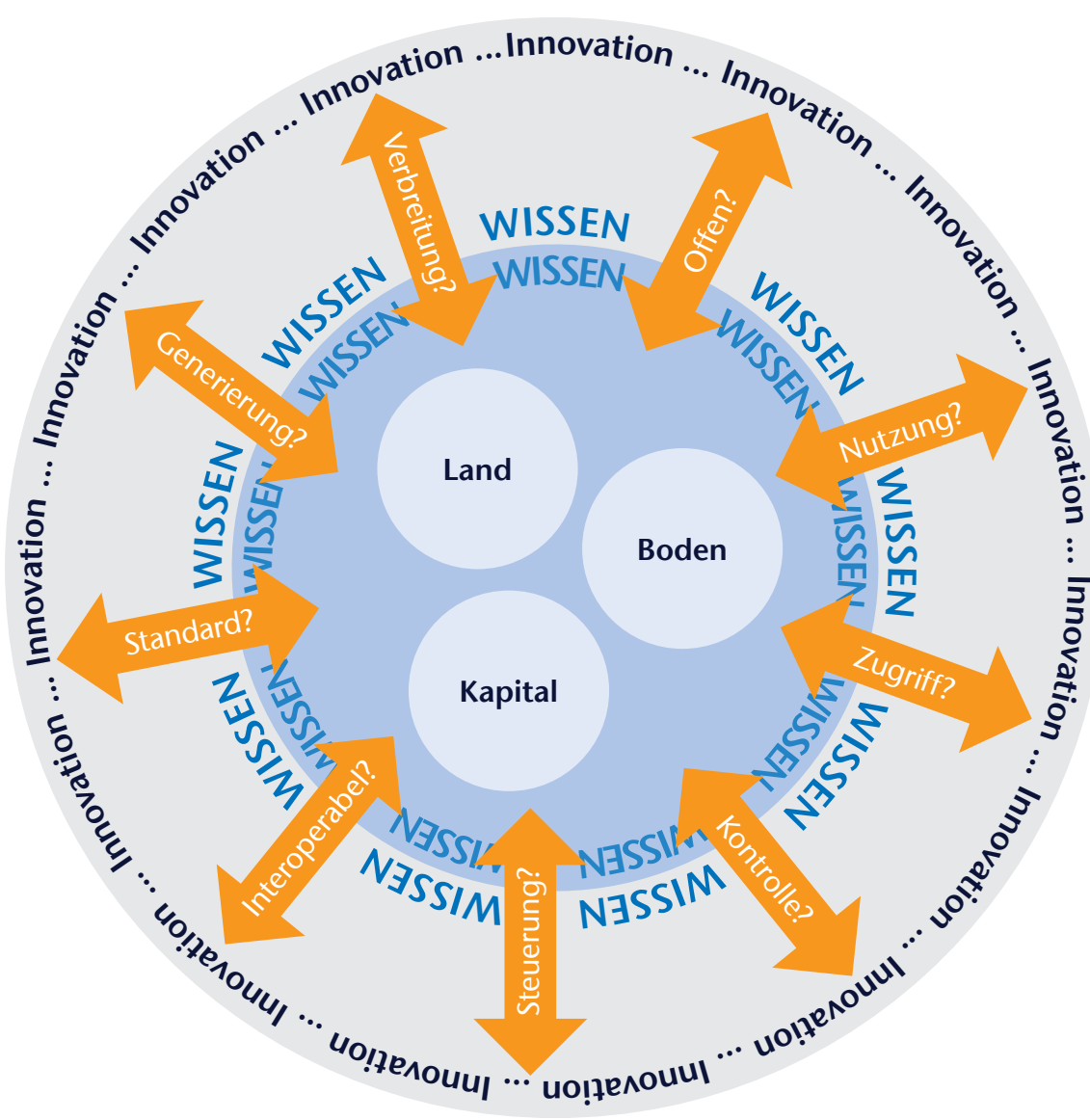

Abb. 1) Die Abbildung skizziert den Bedeutungszuwachs von Wissen für unsere Gesellschaft in Hinblick auf zukünftige Innovationen und die sich aktuell ergebenden Fragestellungen.

Der rechtliche und ökonomische Kampf um die Kontrolle über die Produktion und Distribution von Wissen wird bereits ausgefochten. Traditionell geschieht dies über das Recht, d. h. durch gewerbliche Schutzrechte rund um das geistige Eigentum. Eine große Rolle spielen aber auch die technologischen Möglichkeiten der dezentralen Verbreitung von Wissen und Informationen durch das Internet, die diesen Kampf anheizen und verstärken (Drossou et. al 2006: 1). Ausgangspunkt unseres Beitrags ist die Annahme, dass die Kommunikationsinfrastruktur des Internets die Auseinandersetzung um die Kontrolle über das Wissen nicht nur anheizt, sondern diese ähnlich wie das Recht aktiv steuert. Auf diesen Zusammenhang hat bereits der amerikanische Rechtsgelehrte Lawrence Lessig mit seiner The- se vom Code als Recht (Code is law) verwiesen. Er meint damit, dass der Code als Regulationsinstanz wie Recht, Markt oder soziale Normen eine verhaltenssteuernde Wirkung entfaltet (Lessig 2002). Technische Maßnahmen werden damit zum Instrument vorsätzlicher und zielgerichteter Sozialintervention. Daraus lässt sich folgern, dass sich der "Code“ auch zum wirksamen Steuerungsinstrument im Umgang mit Wissen eignet. Lessig bezieht sich in seiner Argumentation insbesondere auf die Maßnahmen des Kopierschutzes, die bei Erscheinen seines Buches entgegen dem geltenden Recht eine private Kopie legal erworbener Werke verhindert haben. Die aktuell diskutierten Verfahren zur Kontrolle der Nutzung und Verbreitung digitaler Inhalte durch $\mathrm{DRM}^{3}$-Systeme sind ein typisches Beispiel dafür, den Zugriff auf Wissen mittels technischer Maßnahmen zu begrenzen. Der Zugriff auf Wissen wird hier nicht durch Recht, sondern durch Technik reguliert.

Um den Umgang mit Wissen geht es auch im Ansatz des Open Government. Open Government bezeichnet als Sammelbegriff alle Maßnahmen der Regierungen und Verwaltungen, die dazu dienen, das Regierungs- und Verwaltungshandeln transparenter zu machen, die Bürger und Unternehmen stärker zu beteiligen und eine ebenenübergreifende Zusammenarbeit aller Akteure zu ermöglichen. Als wichtiger Schritt in diese Richtung wird ein wesentlich offenerer Umgang mit den Dokumenten und Datenbankinhalten des öffentlichen Sektors gesehen (Open Government Data).

Deutschland folgt dem weltweiten Trend und erklärt Open Government zu einem Ziel seiner IT-Strategie, auch wenn es derzeit unklar bleibt, wie weit die Verwaltungen z. B. mit der Veröffentlichung ihrer Daten gehen können, wollen oder sollen. Welche Daten unter welchen Bedingungen lizenzfrei oder lizenziert veröffentlicht werden und ob die bereitgestellten Daten auch als "offen“ bezeichnet werden können, hängt von verschiedenen Faktoren und Entscheidungen ab. In der vom Bundesinnenministerium (BMI) in Auftrag gegebene Studie „Open Government Data Deutschland“"4, wurden bereits Geldleistungsmodelle für Open Government Data untersucht (Klessmann et al., 2012). Grundsätzlich gilt es zu klären, wie die elektronische Verfüg- und Auffindbarkeit von Open Government Data umzusetzen ist und wie die Nutzungskosten und rechtlichen Rahmenbedingungen zu gestalten sind.

Interoperabilität ${ }^{5}$ und offene Standards gehören zu den Kernthemen der aktuellen Debatte um Open Governement Data. Wir sehen in der Interoperabilität eine der Grundvoraussetzungen für neue Formen sozialer Kooperation, die

\footnotetext{
${ }_{3}^{3}$ Digital Rights Management versucht die Nutzung und Verbreitung digitaler Inhalte durch vergebene, unterschiedlich weit reichende Nutzungsrechte, technisch zu sichern.

${ }^{4}$ Presseerklärung des BMI am 1.8.2012: Die Studie wurde vom Fraunhofer-Institut für Offene Kommunikationssysteme FOKUS erstellt, unterstützt durch das Lorenz-von-Stein-Institut für Verwaltungswissenschaften an der Christian-Albrechts-Universität zu Kiel und der ÖPP Deutschland AG http://www.bmi.bund.de/SharedDocs/Pressemitteilungen/DE/2012/mitMarginalspalte/07/opengovernment.html;jsessionid=B1255FC0E1A0E8C3CC8ABCCB4C21C73F.2_cid239, Zugriff: 16.9.2012.
}

${ }^{5}$ Fähigkeit verteilter Systeme, problemlos und effizient zusammenzuarbeiten und sich auszutauschen. 
auf der technischen Ebene durch den freien Austausch und die Vernetzung von Daten ermöglicht wird. Wir möchten im Folgenden der Frage nachgehen, inwieweit und in welcher Form offene Standards und verteilte Anwendungen als technische Grundlage einer „verteilten Wissensarbeit" wirksam werden. Der Begriff "verteilte Wissensarbeit" lehnt sich an Helmut Willkes Begriff der „Organisierten Wissensarbeit“ an. „Wissensarbeit “ gilt hier als eine Tätigkeit, die Wissen voraussetzt und Wissen erzeugt und sich dadurch auszeichnet, dass es als permanent revisionsbedürftig erachtet und als Ressource betrachtet wird. "Organisierte Wissensarbeit" nutzt den Prozess des Organisierens, um Wissen als Produktivkraft zu entfalten (Willke 2001). Der von uns verwendete Begriff „verteilte Wissensarbeit" bezieht sich auf denselben Wissensbegriff, verlässt aber den Rahmen der abgrenzbaren Organisation und findet auf die kollaborative Arbeit jener Communities Anwendung, die sich ebenfalls der Wissensarbeit widmen.

Verteilte Wissensarbeit kann auch in der öffentlichen Verwaltung helfen, Komplexität zu beherrschen und scheint ideal für einen Ansatz der strategischen Öffnung im Open Government. Hier bietet sich Potenzial, die Verwaltungsorganisation mit ihren Prozessen und Wissensressourcen mit dem Wissen verwaltungsexterner Akteure zu verbinden. Wie auch in der BMI Studie ausgeführt, bildet „der Aspekt der Offenheit [...] einen Ansatz zur Strukturierung von Prozessen und zur Ausrichtung strategischer Entscheidungen“ (Klessmann et al. 2012: 7).

\section{»II. WEBSTANDARDS: OFFENHEIT ALS PRINZIP}

Webstandards sind Spezifikationen des $\mathrm{W}^{3 \mathrm{C}^{6}}$ und dienen der Implementation von Webtechnologien und -anwendungen. Sie schaffen die gemeinsame Grundlage für die Sicherung der Konsistenz und der Interoperabilität des Internets. Sie sorgen somit dafür, dass

\footnotetext{
${ }^{6}$ World Wide Web Consortium

${ }_{7}$ Standards and the Future of the Internet, Geneva 2008, http://www.openforumeurope.org/library/geneva/declaration/ manifesto-with-logos-final.pdf, Zugriff: 10.01.2011).

${ }^{8}$ Application Programming Interface
}

Informationen in heterogenen Soft- und Hardwarewelten ausgetauscht werden können und bilden die Grundlage, die das Internet als freie Kommunikationsinfrastruktur auszeichnet. Interessanterweise lassen sich Standards selbst als Wissen begreifen. Ebenso wie alle anderen Formen des Wissens können auch die Webstandards als Gemeingut frei verfügbar sein oder als "geistiges Eigentum" dem Wissensschutz unterliegen.

Es spricht einiges dafür, dass die große Durchschlagskraft und der Erfolg des Internets in der Offenheit und in der freien Verfügbarkeit seiner Standards liegen und nicht in ihrem "Schutz“. Tim Barners-Lee, der Schöpfer des WWW und Begründer des W3C, beschreibt die Bedeutung „offener" Standards am Beispiel von HTML:

"It was the standardisation around HTML that allowed the web to take off. It was not only the fact that it is standard but the fact that it is open and royalty-free. If HTML had not been free, if it had been proprietary technology, then there would have been the business of actually selling HTML and the competing JTML, LTML, MTML products. Because we would not have had the open platform, we would have had competition for these various different browser platforms but we would not have had the web. We would not have had everything growing on top of it. So I think it very important that as we move on to new spaces [...] we must keep the same openness we had before. We must keep an open internet platform, keep the standards for the presentation languages common and royalty-free. So that means, yes, we need standards, because the money, the excitement is not competing over the technology at that level. The excitement is in the businesses and the applications that you built on top of the web platform."

Es gibt zwar keine allgemeingültige Definition offener Standards, aber eine Vielzahl von Vorschlägen, die gemeinsam haben, dass sie zwei Dinge postulieren:
1. Offene Standards müssen öffentlich und transparent unter Einbeziehung aller beteiligten Akteure entwickelt werden.

2. Offene Standards müssen uneingeschränkt zugänglich sein.

Offene Standards stehen somit selbst für Transparenz, Offenheit und Kontrollverzicht bzw. für eine Kontrolle, die durch alle beteiligten Akteure gesichert wird. Sie sind eine wichtige Basis für die freie Distribution von Wissen und der kollaborativen Wissensarbeit in Communities.

\section{» III. DIE TECHNISCHE EBENE: FREIHEIT UND OFFENHEIT ALS GRUNDLAGE FREIER WISSENSDISTRIBUTION}

Offene Standards spielen eine bedeutende Rolle für die Interoperabilität von Daten und Anwendungen. Sie erschließen damit gleichzeitig neue Wege der Wissensdistribution und -generierung, die durch räumlich verteilte Anwendungen und leichtgewichtige Programmiermodelle geschaffen werden. Zu diesen Weiterentwicklungen des Internets gehört u. a. das Konzept des Webservice, das sich in den letzten Jahren zu einem Paradigma der Informatik entwickelt hat. Die Idee dahinter ist schlicht und sie ist einfach: Komplexe Anwendungen werden nicht zentralisiert auf einem Server ausgeführt, sondern setzen sich aus verschiedenen Komponenten zusammen, die über das Internet verteilt sein können. Die Kommunikation zwischen den Komponenten erfolgt über klar definierte Schnittstellen (API) ${ }^{8}$, die auf Abruf in eine Anwendung eingebunden werden können (Schwenk 2010: 216). Webservices lassen sich in diesem Sinne als vernetzte Maschine-zuMaschine-Kommunikation begreifen, die in der Regel automatisiert ablaufen und deren gemeinsame Sprache auf offenen und herstellerunabhängigen Standards beruht (Zeppenfeld und Finger 2009: 38).

Als innovative Verknüpfung verschiedener Anwendungen setzten Webservices immer zweierlei voraus: einen Paradigmenwechsel im Verständnis der Rolle und Funktion von Software und einen Paradigmenwechsel im Umgang mit 
Daten, Kontrolle und Zugriffsrechten. Der Webpionier Tim O'Reilly beschreibt dies in seinem Aufsatz "What is Web 2.0?" wie folgt:

An die Stelle von Software im Paket treten Services, die ihre Dienste im Internet bereitstellen. Der eigentliche Wert dieser Dienste liegt nicht in den Anwendungen, sondern in den Daten, die hinter den Anwendungen liegen. Die Entwicklung der Anwendungen folgt leichtgewichtigen Programmiermodellen, die lose gekoppelte, d. h. verteilte Systeme erlauben (O'Reilly 2005).

Diese Daten werden anderen Anwendungen über offene Schnittstellen (APIs) verfügbar gemacht. Die Verteilung und der Austausch der Daten erfolgen über einfache Technologien, die Daten zwar bereitstellen, nicht aber deren Verwendung am anderen Ende der Leitung kontrollieren. Der Grundsatz lautet: "Kooperiere ohne zu koordinieren“ (O’Reilly 2005).

In diesem Paradigmenwechsel wird deutlich, dass im Konzept des Webservice gleichsam die Prinzipien der Freiheit und Offenheit wirksam werden. Sie unterscheiden sich damit grundlegend vom Paradigma der monolithischen Software-Architekturen, die alle funktionalen Elemente zu einem homogenen Gebilde zusammenfügen. Dass dieses Konzept den Grundsätzen der Offenheit und Freiheit entgegengesetzt ist, zeigt sich u. a. darin, dass diese Systeme häufig in enger Kopplung an Ressourcen wie Hardware, proprietäre Datenformate und Schnittstellen gebunden sind. Einen Gegensatz dazu bilden Client-Server-Architekturen oder ganz allgemein verteilte Systeme. ${ }^{9}$ Vor diesem Hintergrund lässt sich sagen, dass Offenheit durch den Verzicht auf Kontrolle zu den zentralen Werten dieses Paradigmenwechsels zählt, der den freien Austausch von Daten und Funktionen technisch ermöglicht (Büschenfeldt 2011: 135ff.). O'Reilly formuliert den dabei wirksam werdenden Kontrollverzicht in der Formel „Kooperiere ohne zu koordinieren“: In seiner Argumentation stehen einfache Technologien wie RSS (Really Simple Syndication) oder REST (Representational State Transfer) für das einfache Verteilen und Austauschen von Daten, ohne dass dabei die Freigabe dieser Daten mit dem Anspruch verbunden ist, zu kontrollieren, was am anderen Ende der Leitung geschieht (O'Reilly 2005).

In dieser Art von Offenheit sieht O'Reilly die Voraussetzung für ein wesentliches Merkmal des Web 2.0, der Innovation durch Zusammenbau. Die lose Kopplung verteilter Daten und Funktionalitäten erschließt somit ganz neue Potenziale für Softwareinnovationen, die darauf beruhen, dass es möglich wird, relativ frei auf andere Dienste zuzugreifen und diese mit den eigenen zu vermengen. O'Reilly bezeichnet dieses als "The Right to Remix“. Unter der Bezeichnung „Mashup“ konnte auf diese Weise eine ganze Landschaft unterschiedlicher Anwendungen erblühen, die ihren Mehrwert überwiegend durch importierte Inhalte schaffen, dabei durch die Kombination vieler Dienste reicher werden und dennoch einfach bleiben (O'Reilly 2005, Koch und Richter 2009).

Bei den Anwendungen zeigt sich das innovative Potenzial der gleichrangigen, selbstorganisierten und vernetzten Koordination in den sogenannten Mashups. Der Begriff, der ursprünglich aus der Musikszene stammt, beschreibt die Collage aus verschiedenen Versatzstücken in einem Remix. Als Begriff des Internets stehen Mashups für Medieninhalte, die durch die Rekombination bereits existierender Inhalte gewonnen werden und für Anwendungen, die durch Zusammenbau entstehen. In Mashups verwirklicht sich Tim O'Reillys (2005) Gestaltungsgrundsatz „Gestalte mit Blick auf ,Hackabililty“ und ,Remixability “". Innovationen entstehen durch die Offenheit für überraschende Weiterentwicklungen, die durch den Grundsatz „Kooperiere ohne zu kontrollieren“ verteilte Anwendungen über APIs und offene Standards ermöglichen, die vom ursprünglichen Erfinder gar nicht beabsichtigt oder eingeplant waren (O'Reilly 2005). Für die Innovation durch Zusammenbau stehen exemplarisch

${ }^{9}$ Vgl. dazu IT-Wissen Online Lexikon: http://www.itwissen.info/definition/lexikon/Monolithische-Software-Architektur.html (abgerufen am 20.01.2011) hunderttausende Anwendungen, die mit den offenen Programmierschnittstellen von Google maps, twitter oder Facebook entwickelt wurden.

\section{»IV. DIE ANWENDUNGSEBENE: VERTEILTE WISSENSARBEIT IM NETZ}

Das Potenzial verteilter Funktionalität und Daten bringt genau jene Anwendungen und Vernetzungsoptionen hervor, auf der eine "verteilte Wissensarbeit" in vernetzten Communities möglich wird. Interessant ist hierbei, dass die verteilte Wissensarbeit ohne den Rahmen einer klar abgrenzbaren Organisation auskommt und ihr Potenzial gerade darin liegt, dass sie die Grenzen der Organisation zu überschreiten vermag. Interessant ist ferner, dass sowohl die technische Basis als auch die Kooperationsformen auf der (sozialen) Anwendungsebene auf einem gemeinsamen Prinzip beruhen, das wir in Anlehnung an die Steuerungstheorie Helmut Willkes, als "demokratisches Prinzip“ (Büschenfeldt 2011) bezeichnen. Dieses Prinzip entspricht der heterarischen Organisation und beruht auf dem Grundsatz der "gleichrangigen, selbstorganisierten und dezentralen Koordination" (Willke 1998: 89f.).

Wenn der Austausch zwischen vernetzten Webanwendungen auch automatisiert abläuft, so finden sich im „offenen“ Umgang mit Daten und Programmfunktionen auch hier die Mechanismen der Gleichrangigkeit und Vernetzung wieder. Beides fließt gewissermaßen als Gestaltungsprinzip in technische Komponenten ein. Voraussetzung ist ein "offener" Umgang mit Wissen und Daten. Das gilt - wie bereits erwähnt - für die "offenen" technischen Standards, die für die Konsistenz und Interoperabilität im Netz sorgen, das gilt für die Bereitstellung von Daten und Funktionalität durch Webservices, das gilt aber auch vor allem für das Internet als Programmierplattform. Hier bildet der Grundsatz der Netzneutralität die technische Grundlage einer demokratischen Kommunikationsstruktur dezentraler Vernetzung, die auch als Many-to-many Struktur bezeichnet wird. Umgesetzt wird dieser Grundsatz in der End-to-EndRichtlinie, die vor allem dreierlei verkörpert (Saltzer et. al 1984; Sandwig 2003): 
die Offenheit für alle Nutzer und Nutzungen,

eine polyzentrische (Many-to-many-)Netzwerkarchitektur des Transportsystems

und einen gleichberechtigten, diskriminierungsfreien Zugang für alle Nutzer und Nutzungen.

Das „demokratische Prinzip“ wird in dieser Argumentation zunächst auf der technischen Ebene wirksam und pflanzt sich über das Anwendungsmodell der Webanwendungen auf die Arbeitsweise der Communities fort. Auf der sozialen Ebene werden die Daten der Maschinen zum Wissen der Menschen und führen dort zu jenen Erscheinungen, die unter dem Begriff Social Media bzw. Web 2.0 gerühmt werden. Dazu zählen beispielsweise Phänomene wie die "Demokratisierung" der Medieninhalte durch User-generated-content, die Entfaltung kollektiver Intelligenz oder des Crowdsourcing ${ }^{10}$. Dies wird erst deshalb möglich, weil innovative Webanwendungen ihren Nutzern genau jene Freiheiten einräumen, die notwendig sind, um sich und ihr Wissen zu vernetzen. In dieser, auf Offenheit basierenden, Wissensvernetzung entsteht der Mehrwert offener Daten bzw. des frei verfügbaren Wissens.

Bei den Nutzern der Social Media Anwendungen zeigt sich die Kraft des „demokratischen Prinzips“ nicht nur in Freundschaftsnetzwerken oder im Austausch von Belanglosigkeiten, sondern auch in der ernsthaften und fruchtbaren Wissensarbeit. Jüngere Forschungsarbeiten wie beispielsweise die empirischen Untersuchungen des MIT-Forschers Eric van Hippel bestätigen dies. Seine Untersuchungen identifizieren ausgerechnet in der vermeintlich passiven Nutzergemeinschaft einen neuen Innovatorentypus. Die sozialen Gebilde, die es möglich machen, dass die „Entwicklung, Verbreitung und zum Teil auch die Produktion von Innovationen durch Anwender, für Anwender" erfolgen kann, bezeichnet van Hippel als Anwender-Innovationsnetzwerke. Sie schmälern die Bedeutung der Warenproduzenten, die bislang als Träger der Innovation galten, und machen diese, sofern es sich um Informationsprodukte

Anwender Innovations Netzwerke, Crowd Sourcing, User Generated Content

Social Media
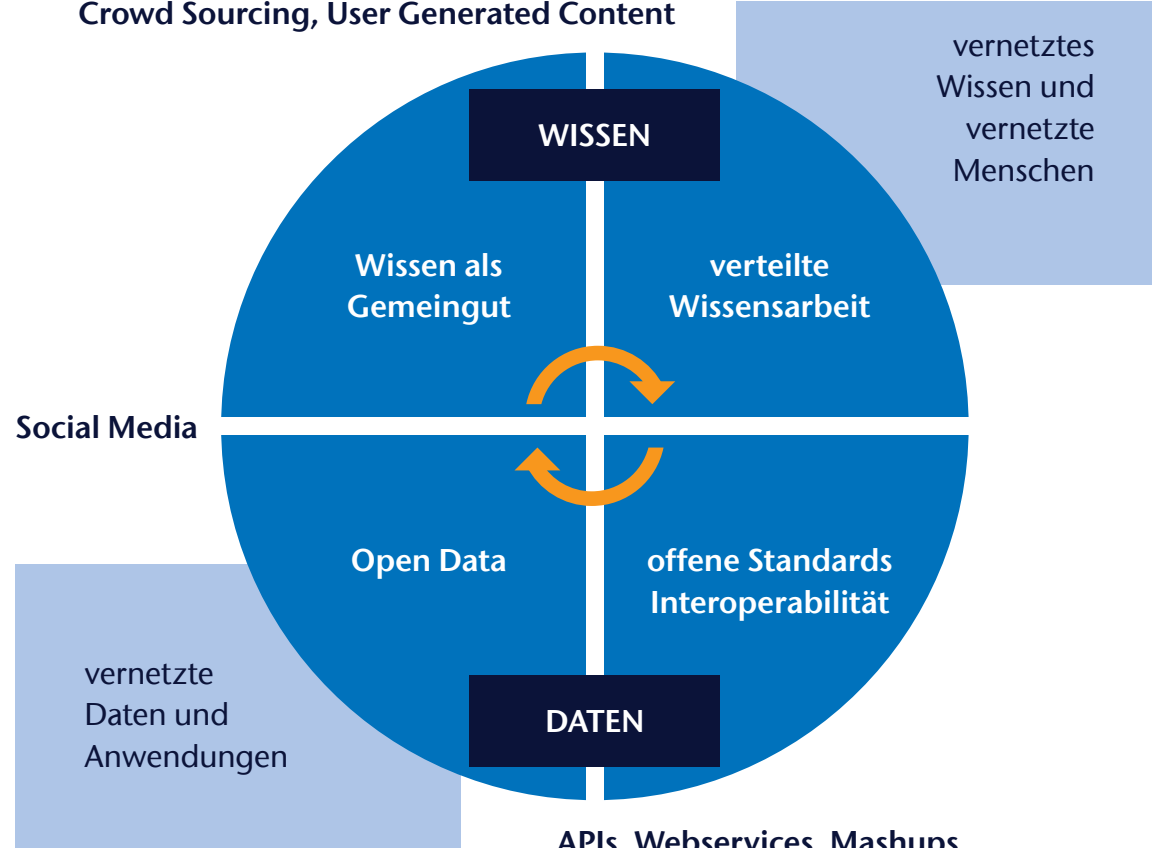

APIs, Webservices, Mashups

Abb. 2) Verteilte Wissensarbeit

handelt, sogar entbehrlich (Hippel 2005). Besonderes Merkmal dieser Netzwerke ist ihre heterarchische Struktur, d. h. ihr horizontaler und vernetzter Aufbau'11. Die Grenzen zwischen den beteiligten Akteursgruppen wie Hersteller und Anwenderlnnen verwischen ebenso wie die Grenzen zwischen Entwicklung und Nutzung der entwickelten Produkte. Alle Beteiligten dieser Netzwerke sind "innovative Anwender" bzw. Anwender als "Selbsthersteller“, die in der Lage sind, Innovationen nicht nur zu konsumieren, sondern auch zu entwickeln, herzustellen und zu verbreiten (Hippel 2005: 453).

Ein geradezu ideales Beispiel, das ebenfalls Gegenstand der Untersuchungen von van Hippel war, sind die Open Source Communities. So findet sich im BasarModell der Open-Source-Entwicklung (Raymond) ebenso wie in der Vision eines Web 2.0 (O'Reilly 2005) die Idee intelligenter, sozialer Vernetzung aktiver NutzerInnen, die in den Softwareentwicklungsprozess als Mitentwicklerlnnen und InnovatorInnen involviert sind (Raymond, 2000; O'Reilly 2005, Hippel 2005). Die Offenheit der Methoden und Praktiken des Basar-Modells bietet damit auch ein erhebliches Potenzial sozialer Innovation durch eine breitere Nutzerbeteiligung. Bezeichnenderweise ist die Entwicklung der bekanntesten Tools des Web 2.0 wie Weblogs und Wikis ebenfalls durch Pionieranwender bestimmt, deren Hauptmotivation ein persönliches Anliegen war und welche die Verstrickung der Anwender-Innovatoren in die Entwicklung ihrer Produkte offenbar werden lässt: sie entwickeln nicht nur, sondern sie nutzen diese auch. ${ }^{12}$

\section{» V. RESÜMEE UND AUSBLICK: UMSETZUNGSOPTIONEN DES DEMOKRATISCHEN PRINZIPS OFFENER STANDARDS IM OPEN GOVERNMENT}

Gleichrangigkeit, Offenheit und eine dialogische Kommunikationsstruktur werden insbesondere durch Technologien ermöglicht, die einen offenen 
Datenaustausch über die Grenzen einer Anwendung hinweg in maschinenlesbarer Form ermöglichen. Die dazu genutzten Standards als auch die verteilten Inhalte müssen idealerweise frei sein (Büschenfeldt 2011: 161), will man einen kreativen Umgang für innovative Anwender fördern und eine Beschneidung nicht vorhersehbarer Innovationen vermeiden. Die Kommission des European Interoperability Framework (EIF) teilt die Ansicht, dass die Verwaltungen offene Standards wegen ihres positiven Effekts auf Interoperabilität verwenden sollten. ${ }^{13}$

Eine Übertragung auf die Diskussion um Open Government Data liegt keineswegs fern, wenn auch gewisse Begrenzungen durch vielfältige, rechtliche Rahmenbedingungen existieren. So gilt „Informationsfreiheit [...] als Element moderner Staatlichkeit und als ein in
Zukunft zunehmend unverzichtbarer Bestandteil eines partizipativen demokratischen Rechtsstaats (Klessmann et al. 2012: 8). Informationsfreiheit bedeutet jedoch nicht nur, dass Bürger sich aus allgemein zugänglichen Quellen oder auf Antrag bei der Verwaltung informieren können, sondern dass „Informationen, Meinungsbildungs-, Abwägungs- und Entscheidungsprozesse“ aktiv offengelegt und die Daten der öffentlichen Hand für Dritte innerhalb und außerhalb öffentlicher Stellen verfügbar sind (Klessmann et al. 2012: 8).

Laut der BMI-Studie soll das Auffinden geeigneter Datensätze sowie die Verarbeitung durch Akteure aus Zivilgesellschaft, Wissenschaft, Wirtschaft und Verwaltung auf höchstem Niveau und so effizient wie möglich gestaltet werden (Klessmann et al. 2012: 9). Dazu sei

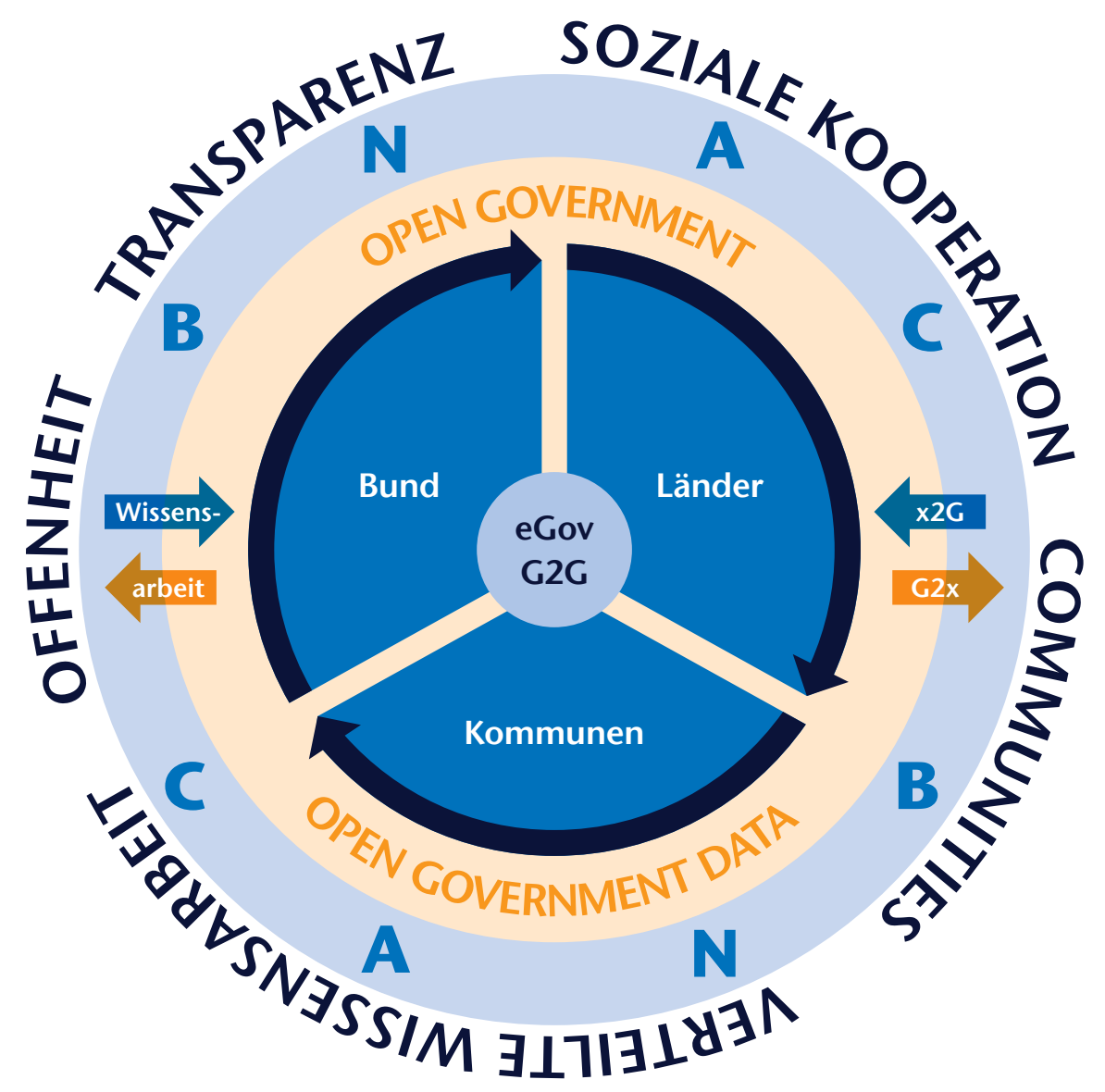

Abb. 3) Die Abbildung skizziert das G2x-Beziehungsgeflecht für soziale Kooperation unter dem Einfluss von Open Government. $G=$ Government, $C=$ Citizen, $A=$ Agent, $N=$ Non-Profit Organisation, $B=$ Business.

${ }^{12}$ So zählten zu den ersten Bloggern eben auch Menschen wie David Winer, die maßgeblich an der Entwicklung der Weblogs und ihrer Vernetzungstechnologien140 mitgewirkt haben. Die erste Wiki-Software wurde von dem Programmierer Ward Cunningham geschrieben, der ein Tool für den Austausch von Code-Fragmenten mit anderen Programmierern suchte.

${ }^{13}$ Linux Magazin, EIF fordert quelloffene implementierbare Standards,

http://www.linux-magazin.de/NEWS/EIF-2-fordert-quelloffen-implementierbare-Standards, Zugriff 20.10.2010.

einerseits das Datenangebot fortwährend auszubauen, andererseits sollen den Datenbereitstellern und -nutzern entsprechende Werkzeuge an die Hand gegeben werden - wie diese und damit die dahinterliegende Technologie prinzipiell aussehen werden, wird in naher Zukunft zu entscheiden sein. In der BMIStudie werden hinsichtlich der bis 2013 angestrebten föderalen Open-Government-Data-Plattform Empfehlungen für die Metadaten- und Datenstandards ausgesprochen. Empfehlungen für Formate und Vorgehensweisen, die eine Vereinheitlichung befördern, sollten noch ausgearbeitet werden. Sicherheitsrelevante Aspekte werden zwar klassifiziert, aber noch ohne einschränkende Empfehlung aufgelistet. Für die Plattformtechnologie selbst wird das Portalsystem Liferay vorgeschlagen, das auf Java basiert und mit einer Reihe von Internet WWW Standards kompatibel ist, unter anderem: offene Standards für Webinhalte, Portlets, Webservices und Weboberflächen-Technologien.

Die BMI-Studie zeigt in ihrer Langfassung auch detaillierter auf, dass offene Verwaltungsdaten für die verschiedenen Zielgruppen unterschiedliche Chancen bieten und vor unterschiedliche Herausforderungen stellen. Die Offenlegung der Datenbestände und die Nutzung bzw. Weiterverarbeitung durch sogenannte Dritte könnte auch Effekte wie Qualitätssicherung und -steigerung mit sich bringen. Die öffentliche Verwaltung wird allerdings nicht den steigenden Qualitätsanforderungen ihrer mündigen Bürger gerecht werden und vom Wissen verwaltungsexterner Akteure profitieren können, wenn Kontrollen und eingeschränkte Zugriffe sich bei der Umsetzung von Open Government als Hindernisse erweisen. Darüber hinaus ist auch das Wissen der eigenen Mitarbeiter/innen eine Ressource, die persönlich entwickelt und eingesetzt werden kann oder eben nicht. Hier könnten Strukturveränderungen über Kontextsteuerung helfen, zu einer stärkeren und motivierenden Selbstorganisation zu gelangen (Scholl et al. 2011). Daher wird Open Government mit offenen Standards zu Veränderungen in der Wissensarbeit auch innerhalb der öffentlichen Verwaltungsstruktur führen müssen, um „verteilte Wissensarbeit“ mit verwaltungsexternen Akteuren zu 
fördern, so dass gleichrangige Partizipation und offene Transparenz in einer dialogischen Kommunikationsstruktur resultieren können.

\section{LITERATUR}

Büschenfeldt, M. (2011): Die Zukunft elektronischer Demokratie: Die Bedeutung des demokratischen Prinzips in softwaretechnischen Konzepten und der elektronische Demokratie als Anwendungsdomäne der Softwareentwicklung, E-Dissertation, Bremen.

Clases, Ch., Wehner, Th. (2002): Handlungsfelder im Wissensmanagement, in W. Lüthy, E. Voit \& T. Wehner (Hrsg.), Wissensmanagement-Praxis-Einführung, Handlungsfelder und Fallbeispiele, vdf, Zürich.

Drossou, O., Krempl, St., Poltermann, A. (2006): Die wunderbare Wissensvermehrung - Wie Open Innovation unsere Welt revolutioniert, 1. Aufl., Heise, Hannover.

Grassmuck, V. (2004): Freie Software zwischen Privat- und Gemeineigentum; 2. Aufl. Bundeszentrale für politische Bildung; Bonn.

Hippel, E. van (2005): Anwender-Innovationsnetzwerke - Hersteller entbehrlich. In: Lutterbeck, B.; Gehring, R. A.; Bärwolff, M. (Hrsg.): Open Source Jahrbuch; Lehmanns Media; Berlin, 450-461.

Klessmann, I., Denker, Ph., Schieferdecker, I., Schulz, S.E. (2012): Open Government Data Deutschland - Kurzfassung der Studie zu Open Government in Deutschland im Auftrag des Bundesministeri-um des Innern (BMI), Berlin, PDF

Koch, M.; Richter, A. (2009): Enterprise 2.0 - Planung, Einführung und erfolgreicher Einsatz von Social Software in Unternehmen; 2. Aufl. Oldenbourg Wissenschaftsverlag; München.

O'Reilly, T. (2005): Was ist Web 2.0? Online-Publikation; http://www.oreilly.de/artikel/web20_trans.html (abgerufen am 16.07.2008).

Saltzer, J., Reed, D., Clark, D. (1984): End-to-End Arguments in System Designs; ACM Trans. Comp. Sys; 2 (4), 277-288; http://web.mit.edu/Saltzer/www/publications/ endtoend/endtoend.pdf (abgerufen am 10.08.2009).

Sandvig, C. (2003): Shaping Infrastructure and Innovation on the Internet: The End-to-End Network that isn't it. In: Guston, D., Sarewitz, D. (Hrsg.): Shaping Science and Technology Policy: The Next Generation of Research; University of Wisconsin Press.;Madison; draft version; http: \www.spcomm.uiuc.edulcsandvig \research $\backslash$ Communication_Infrastructure_and_Innovation.pdf; (abgerufen am: 25. 09.2006).

Scholl, M., Büschenfeld. M. (2011): From Planning Proces to Self-Organisation - Can context control help ensure the success of IT projects in public administration? EGOV 2011, August/September 2011 in Delft, Niederlande. Joint Proceedings of Ongoing Research and Projects of IFIP EGOV and ePart, Trauner Verlag, 117-124.

Schwenk, J. (2010): Sicherheit und Kryptographie im Internet; Vieweg+Teubner; Wiesbaden; http://www. springerlink.de/content/u259374530106m05/ (abgerufen am 05.01.2011)

Willke, H. (2001): Systemisches Wissensmanagement, 2. Aufl., Stuttgart.

Zeppenfeld, K., Finger, P. (2009): Serviceorientierte Architektur mit WebService; in: SOA und Web-Services; Informatik im Fokus; Springer; Berlin Heidelberg, 69-86, http://dx.doi.org/10.1007/978-3-540-76991-0_4.

\section{AUTOREN}

Dr.-Ing. Maika Büschenfeldt

Projektleiterin „Neue Wege, neue Chancen zum lebenslangen Lernen“" (gefördert aus Mitteln des Europäischen Sozialfonds und des Landes Brandenburg)

FB Wirtschaft, Verwaltung und Recht, TH Wildau [FH]

Telefon: +49 (0) 3375 / 508-379

maika.bueschenfeldt@th-wildau.de

Prof. Dr. rer. nat. Margit Scholl

Verwaltungs- und Wirtschaftsinformatik

FB Wirtschaft, Verwaltung und Recht

TH Wildau [FH]

Telefon: +49 (0) 3375 / 508-917

margit.scholl@th-wildau.de

www.th-wildau.de/scholl 